\title{
Classification of soils developed from bottom lake deposits in north-eastern Poland
}

\author{
Andrzej Łachacz ${ }^{1 *}$, Szymon Nitkiewicz ${ }^{2}$ \\ ${ }^{1}$ University of Warmia and Mazury in Olsztyn, Faculty of Agriculture and Forestry, Department of Soil Science and Microbiology, Pl. Łódzki 3; \\ 10-727 Olsztyn, Poland; ORCID iD: https://orcid.org/0000-0003-2902-2767 \\ ${ }^{2}$ University of Warmia and Mazury in Olsztyn, Faculty of Technical Sciences, 11 Oczapowskiego St.,10-710 Olsztyn, Poland, School of Medicine, \\ Collegium Medicum, 30 Warszawska St., 10-082 Olsztyn, Poland; ORCID iD: https://orcid.org/0000-0002-2424-4670
}

* prof.dr hab. Andrzej Łachacz, andrzej.lachacz@uwm.edu.pl

Received: 16.02.2021

Accepted: 30.07.2021

Associated editor: C. Kabała

\section{Keywords}

Soil classification

Gyttja soils

Polish Soil Classification

WRB, Mazurian Lakeland

Sand cover cultivation of fens

\begin{abstract}
The aim of the study was to test the suitability of the 6th edition of Polish Soil Classification (PSC6, 2019) for reflecting the typical features of gyttja soils in comparison with the previous editions of PSC, namely PSC4 (1989) and PSC5 (2011), and the newest version of Word Reference Base for Soil Resources (IUSS, 2015). On the basis of described morphology and determined properties, soils were classified according to different soil classifications. In total, 25 soil profiles were investigated, of which 18 developed from bottom lake deposits and 7 from lakeshore deposits. Based on the presence of three main components of lake deposits: organic matter (determined as loss-on-ignition), calcium carbonate, and mineral non-carbonate fraction, several kinds of limnic materials were identified according to PSC6. Soils developed from deep lake deposits unaffected by the murshing process were classified (PSC6) as gyttja soils, and as murshic gyttja soils when the upper layer was composed of gyttja mursh. Some classification problems arose when soils developed from shallow gyttja deposits located on the former lake shore, and their top layer was affected by deposition of colluvial materials from adjacent slopes. In several soil profiles, the top layer developed from the admixture of sand deliberately deposited by man on the gyttja surface in order to improve soil properties. In Łąki Dymerskie gyttja land, this sandy top layer is 31-39 cm thick and there are written reports on amelioration of this site by sand cover cultivation (Rimpau method, Sanddeckkultur) carried out since 1885. At present, this layer contains 3-20\% SOM and fulfils the requirements of the arenimurszik horizon specified in PSC (2019). The newest edition of PSC enables the user to achieve detailed classification of such soils by using a soil variety, namely the heaped soil (in Polish - nasypowe). According to WRB (2015), such soils are classified mostly as Hypereutric Rheic Drainic Histosols (Limnic, Nechic, Areninovic) ones and Eutric Gleysols (Endoarenic, Drainic, Humic, Limnic, Nechic). Generally, the taxonomic position of soils studied was more detailed according to PSC6 in relation to the occurrence of various soil strata and different soil materials when compared to the previous versions of PSCs. It should be stated that the precision achieved when using PSC6 to classify gyttja soils was similar to that obtained with WRB (2015).
\end{abstract}

\section{Introduction}

The disappearance of lakes is a natural process in the evolution of water bodies. It occurs when the water level in a lake is lowered due to reduced water influx or the accumulation of sediments in the basin, which can be imported from the catchment or produced in situ through various processes taking place in the lake water as well as the death of aquatic organisms. The rate of this process depends on the type of a lake, and particularly its depth, geological structure of the catchment basin, development of the hydrological network, climate and plant cover. Human interference is another significant factor directly affecting the transformation of lakes (Uggla, 1964,
1971; Tobolski, 2000; Berglund and Berglund, 2010; Choiński et al., 2012; Stankevica et al., 2016; Kruczkowska et al., 2021).

Most peatlands in the Masurian Lakeland have evolved in the process of terrestrialisation of lakes, therefore they are underlain by lacustrine deposits. Considering the ongoing process of peat murshing and mineralisation of organic matter, it can be suspected that in the future, due to the peat superficial layers becoming shallower, the exposed layers of bottom sediments will be mixed with surface organic formations and will undergo soil-forming processes. Thus, the surface area of soils formed from bottom lake deposits will continue to grow (Chmieleski and Zeitz, 2006; Lemkowska and Sowiński, 2018). 
In the second half of the 19th century and early 20th century, the territory of former East Prussia (nowadays the region of Warmia and Mazury) turned into a site of intensive hydroengineering works consisting of the artificial withdrawal of water from lakes. River beds were widened and deepened, drainage canals and ditches were dug and water pump stations were built. As a result, the water level in lakes declined, in some areas by as much as a few metres. Some lakes either diminished in surface area or disappeared completely. Drained peatlands around lakes as well as bottoms of dried lakes were put to agricultural use, having been converted to grassland (Uggla, 1971; Ilnicki, 2002; Piaścik et al., 2003; Choiński et al., 2012).

This rapid interruption of the natural evolution of water bodies resulted in the formation of gyttja land - a specific type of wetland, where layers of bottom deposits (gyttja) lie near the surface or else are covered by a shallow layer of peat or other material (e.g. colluvial). The thickness of bottom deposits varies greatly, ranging from several centimetres to several metres (Uggla, 1971; Ilnicki, 2002; Łachacz et al., 2009). The lack of experience in the management of such land gave rise to problems and often surpassed the technical capabilities of the land owners. The specific character of gyttja sediments and changes in its hydration caused either severe shrinkage and formation of cracks or the expansion (swelling), uplift of the surface and clogging of drainage devices (Uggla, 1971; Uggla et al., 1972). Being a highly hydrated colloid, organic gyttja filled the ditches and slowed down the operation of drainage system. For this reason, the same gyttja sites were meliorated repeatedly. Many water bodies could not be drained at all while in others, apparently drained successfully, water resurfaced after a few years and lakes reappeared. Attempts to convert gyttja land to grassland most often failed to yield expected outcomes. Wherever people managed to create meadows, there is now wet and muddy land, a site of secondary bogging. Lack of maintenance of drainage systems resulting in their inferior efficiency and progressive silting of ditches led to the restoration of water bodies with hydrophilic plants and littoral belts of reeds. Nowadays, most gyttja lands are excluded from agricultural production as they are extremely difficult to be used for farming. On the other hand, many of these gyttja sites display high natural values (Piaścik et al., 2003; Łachacz et al., 2009; Łachacz and Pisarek, 2018; Choiński et al., 2012). However, it should be borne in mind that some gyttja lands, especially developed on mixed deposits with a higher share of the mineral component (clay, calcium carbonate) are used for agricultural purposes, as good and moderately good arable land. The agricultural use is also continued on edges of gyttja sites, including ones developed from organic gyttja (Uggla, 1971, 1976; Lemkowska and Sowiński, 2018).

Most of the current classification systems developed for soil science and applied to categorise bottom lake sediments (gyttjas) are based on the determination of three principal components: organic matter (usually determined as weight loss-on-ignition), calcium carbonate, and non-calcareous mineral fraction (expressed together as crude ash). In Poland, such divisions have been proposed by Okruszko (1976), Markowski (1980) and Ilnicki (2002). These classification systems are similar and share the agenetic character (Tobolski, 2000). The adoption of three major classification parameters resulted in a triple structure, typical of all gyttja division systems based on Lundquist's classification (1927).

Due to the specific properties of the bottom lake sediments, the soils that develop therefrom present several characteristics that distinguish them from peat soils, the fact that should be reflected in their classification. The specific nature of gyttja soils is also a consequence of the co-occurrence of carbonate and noncalcareous (silicate) mineral particles and organic matter, most of which appear as colloidal fractions. Non-dehydrated gyttja is therefore a gel, i.e. a semi-fluid gelatinous mass. While drying, gyttja, particularly organic one, shrinks irreversibly. This is associated with the subsidence of gyttja deposits as well as the formation of fissures and polygonal microrelief. When drying up, organic gyttja splits into layers of plate and laminate aggregates, as well as becoming extremely hydrophobic, which has an adverse effect on air and water conditions in soils (Uggla, 1968, 1971; Uggla et al., 1972; Chmieleski and Zeitz, 2006; Papierowska et al., 2009).

The bottom sediments of drained lakes are exposed to impact of the atmosphere and biosphere as well as undergoing soil-forming processes, converting into subareal soils (Uggla, 1962, 1971; Okruszko, 1976). Organic and mixed gyttja, like all soil materials accumulated under anaerobic conditions, are subjected to murshing processes when dehydrated (Uggla, 1962). In Poland, the most common gyttja soils are the ones developed from detrital gyttja. These soils are of little value for agriculture and are most often categorised as wasteland. Calcareous gyttja soils, present in the form of so-called lacustrine chalk or meadow limestone, are less common, and these compose specific soils referred to as post-lacustrine or Quaternary rendzinas (Uggla, 1976; Meller, 2006; Jarnuszewski and Meller, 2018, 2019).

Another reason why soils developed from lake bottom sediments or with their contribution achieve a distinguishing set of features, beside the differences arising from the chemical composition of gyttja, is the presence of thin layers of limnic, peat and peat-like as well as colluvial materials near the shores of dried lakes (Mendyk et al., 2016; Markiewicz et al., 2017). This is particularly true about small water bodies with a changeable water level, such as ponds, including water mill ponds (Mendyk et al., 2015).

The purpose of this article has been to compare the taxonomic position of soils of three gyttja sites located in the Masurian Lakeland, according to consecutive versions of the Polish Soil Classification system: PSC4 (1989), PSC5 (2011), PSC6 (2019), and the World Reference Base for Soil Resources (IUSS Working Group WRB, 2015).

\section{Study area and methods}

Three vast, typical gyttja lands situated in the Masurian Lakeland have been selected for the research (Solon et al., 2018) (Fig. 1). Łąki Staświńskie gyttja land (ca 700 ha) lies in the southeastern part of Giżycko District, the mesoregion of the Great Masurian Lakes Region (Uggla, 1968). Gązwa gyttja land (104 ha) is located in Mrągowo District, the mesoregion of Mrągowo 


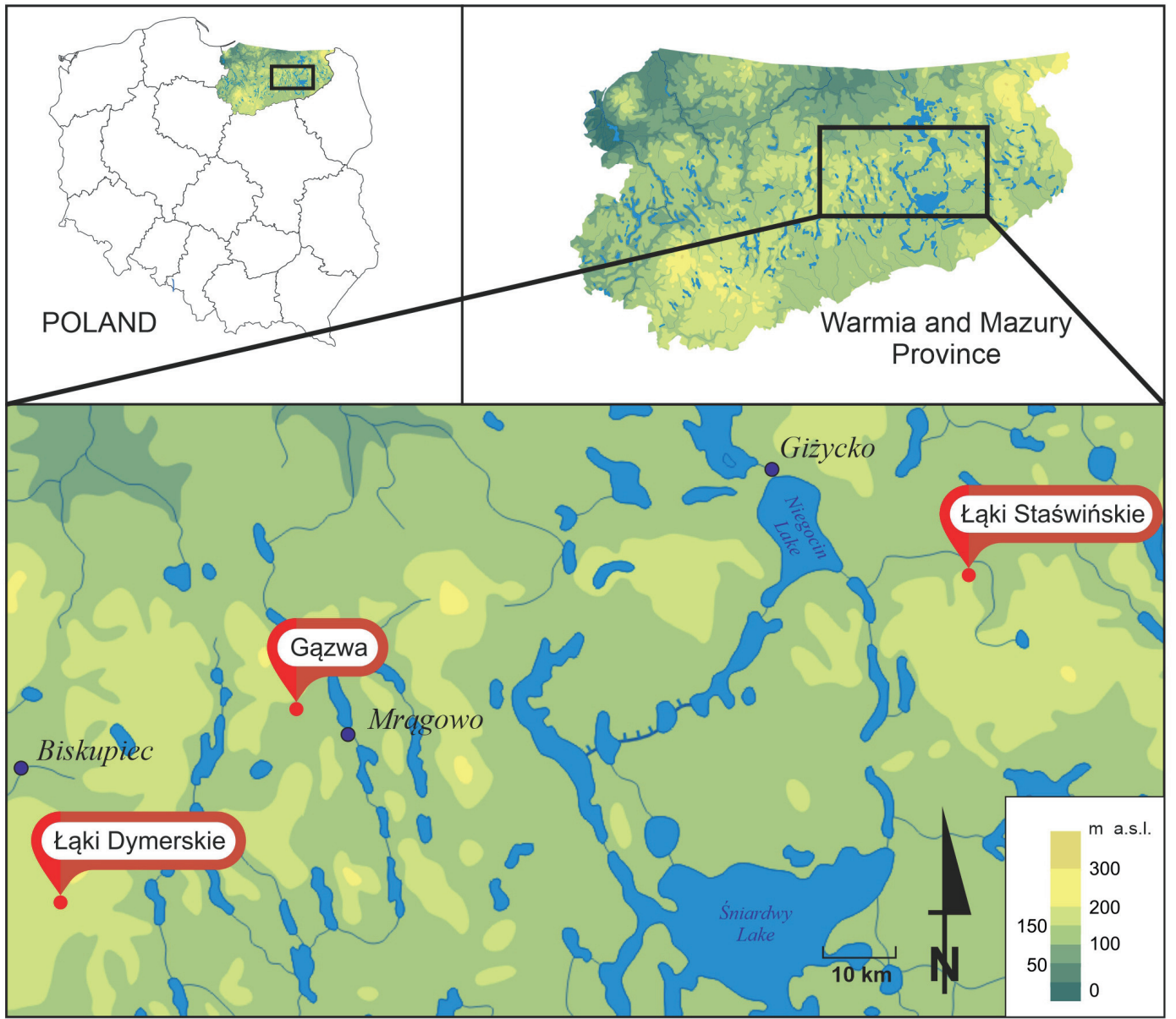

Fig. 1. Location of the studied gyttja sites

Lakeland. Łąki Dymerskie gyttja land (242 ha) is situated in Olsztyn District, in the western part of the mesoregion the Mragowo Lakeland (Choiński et al., 2012; Łachacz and Pisarek, 2018). The gyttja lands included in this study share certain characteristics, namely they were all formed as a result of artificial draining of lakes in the second half of the 19th century, and the draining was carried out using pump set-ups. Afterwards, they were used as meadows until World War Two. After the war, as the maintenance of the drainage systems was neglected, they were undergoing a gradual process of turning into a swampy area again until vast patches in the central parts of these lands were overgrown with reeds (Łachacz et al., 2009).

Twenty-five soil pits in 6 transects were made on the analysed gyttja lands, which was sufficient to characterise the spatial diversity of the soils. Efforts were made to maintain similar distribution of the soil pits on all locations: from mineral soils at the edge to organic ones towards the centre of the former lake, with the transects situated perpendicularly to the main drainage canal. The detailed description of the analysed gyttja sites, including the plant cover and chemical properties of the soils, can be found in our previous paper (Eachacz et al., 2009). The focus of this article is on problems encountered in the classification of soils formed from bottom lake deposits.

The studied soils were described in field, when the soil colour was determined in moist samples, and then in laboratory, by applying Munsell colour charts to dry soil samples (Oyama and Takehara, 1992). Collected samples were air-dried at room tem- perature, visible roots and other living plant remnants were removed manually, the soil was gently crushed with a rolling pin, and sieved through a $2 \mathrm{~mm}$ steel sieve. All the laboratory analyses were made on fine earth material (the fraction $<2 \mathrm{~mm}$ ). The following properties were determined in the sampled material: loss-on-ignition in a muffle furnace at a temperature of $550^{\circ} \mathrm{C}$, the content of calcium carbonate (equivalent) by the Scheibler volumetric method, soil $\mathrm{pH}$ by the potentiometric method in $1 \mathrm{~mol} \cdot \mathrm{dm}^{-3} \mathrm{KCl}$ and in distilled water (soil-to-solution ratio of 1:2.5). Soil texture in mineral soil materials was determined using a hydrometer and sieving methods (van Reeuwijk, 2002; PSC6, 2019). Loss-on-ignition (LOI) was assumed to approximate the organic matter content (SOM). The content of mineral noncarbonate fraction was calculated based on LOI and the content of calcium carbonate. It was assumed in this study that $12 \% \mathrm{C}_{\text {org. }}$ corresponds to $20 \%$ SOM.

\section{Results and discussion}

\subsection{Division of bottom lake deposits}

The soils found in the basins of artificially drained lakes had developed from bottom sediments. The current classification of Polish soils (PSC6), distinguishes limnic materials, among diagnostic soil materials, which consist of organic gyttjas, calcareous gyttjas, meadow limestone (marl) as well as limnetic and telmatic 
muds. Figure 2 illustrates the division of bottom lake deposits according to PSC6. Additionally, at the tip of the triangle there is a field identified as lacustrine clay (clay gyttja). These terms have been presented in the divisions of bottom lake deposits previously used in Poland (Okruszko, 1976; Markowski, 1980; Ilnicki, 2002). However, PSC6 classifies such mineral formations as fluvic materials rather than limnic materials, considering the fact that their sedimentary genesis proceeded with no significant contribution of living organisms, which is evidenced by a low content of organic matter $\left(<12 \% \mathrm{C}_{\text {org }}\right.$ ) and its non-coprogenous character. Thus, figure 2 shows the division of bottom lake deposits comprising limnic materials (organic gyttjas, calcareous gyttjas, meadow limestone) and lacustrine clay (clay gyttja) not counted to limnic materials but to fluvic materials. The division constructed this way and visualised in a triangle contains bottom lake deposits, widespread in Poland, and the additional inclusion of lacustrine clay (as fluvic material) means that all fields in the triangle are filled and described.

The classification system of limnic materials accepted in Poland (Kabała et al., 2019) directly draws on earlier divisions applied in Poland (Okruszko, 1976; Markowski, 1980; Ilnicki, 2002). It is a modification of the most popular division developed by Markowski (1980). However, the Markowski division has certain flaws, such as it is not exhaustive enough and its projection onto an equilateral triangle shows some blank (undescribed) areas, which was demonstrated in an earlier paper (Łachacz et al., 2009). The division of limnic materials comprises three principal
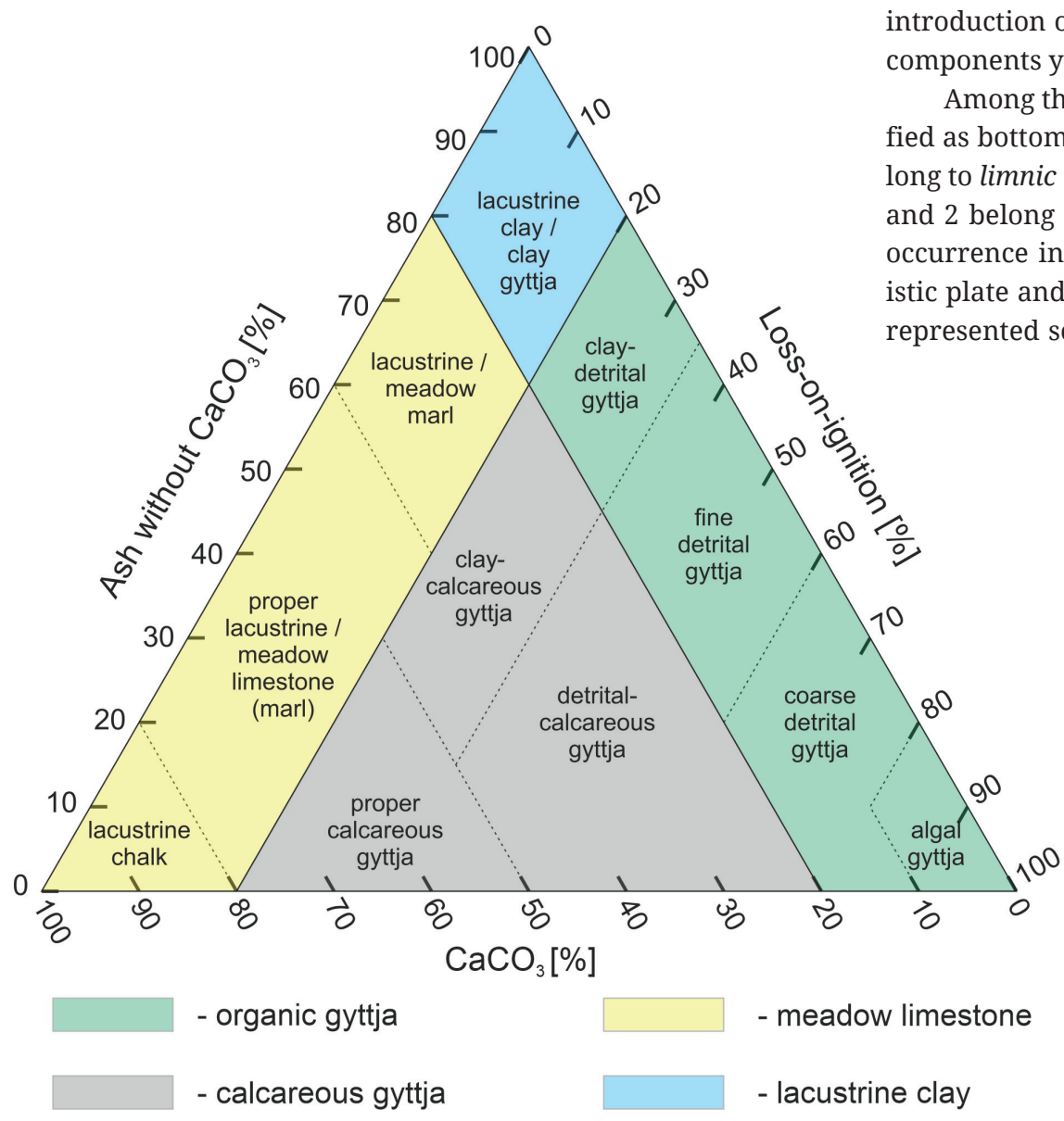

- meadow limestone

- lacustrine clay divides (Fig. 2), such as organic gyttjas, calcareous gyttjas and meadow limestone (or marl), which are then divided into specific types. Organic gyttja contains $\geq 12 \%$ of organic carbon and $<20 \%$ of $\mathrm{CaCO}_{3}$, calcareous gyttja contains $\geq 12 \%$ of organic carbon and $\geq 20 \%$ of $\mathrm{CaCO}_{3}$, meadow limestone (marl) contains $<12 \%$ of organic carbon and $\geq 20 \%$ of $\mathrm{CaCO}_{3}$. The latter type, based on the content of $\mathrm{CaCO}_{3}$, is subdivided into lacustrine/meadow marl, proper lacustrine limestone/meadow limestone (marl) and lacustrine chalk (Kabała et al., 2019). It is therefore possible to distinguish facies formed in an aquatic environment (lake) from ones developed in a terrestrial environment (meadow), although in many cases the identification is a challenge unless special tests are performed (Prusinkiewicz and Noryśkiewicz, 1975; Tobolski, 2000; Freytel and Verrecchia, 2002).

The division adopted in the PSC6 allows the classification of bottom sediments based on field organoleptic observations, followed by detailed laboratory tests. The identification of limnic materials needs to include the observation of their mechanics when pressed (Markowski, 1980). Organic gyttja in wet conditions shows rubber-like consistency. It is resilient, i.e. able to spring back to previous shape after being compressed (Kabała et al., 2019). After drying, organic gyttja disaggregates into lamellae. Gyttjas with a lower content of organic matter (e.g. calcareous gyttjas) display weak resilience when moist, proportionally to their lower amount of organic matter. When organic matter is determined in terms of the loss-on-ignition (LOI), then all the three component parts add up to $100 \%$. Determination of organic matter based on the content of organic carbon requires the introduction of a conversion factor so that the sum of the three components yields $100 \%$.

Among the analysed soil horizons, 44 samples can be classified as bottom lake deposits (Table 1, Fig 3). Among them 42 belong to limnic materials as specified in PSC6 (Kabała et al., 2019), and 2 belong to fluvic materials (lacustrine clay). Based on the occurrence in the top layers of soil profiles and the characterstic plate and laminate structure, it was stated that 15 samples represented soil materials undergoing the process of murshing 
Table 1, continue

\begin{tabular}{|c|c|c|c|c|c|c|c|c|c|c|c|}
\hline \multirow{2}{*}{$\begin{array}{l}\text { Depth } \\
\text { [cm] }\end{array}$} & \multirow{2}{*}{$\begin{array}{l}\text { Soil } \\
\text { horizon* }\end{array}$} & \multirow{2}{*}{$\begin{array}{l}\text { Bottom } \\
\text { lake } \\
\text { deposit } \\
\text { No.** }\end{array}$} & \multirow{2}{*}{$\begin{array}{l}\text { LOI } \\
{[\%]}\end{array}$} & \multirow{2}{*}{$\begin{array}{l}\mathrm{CaCO}_{3} \\
{[\%]}\end{array}$} & \multicolumn{2}{|l|}{$\mathrm{pH}$} & \multicolumn{2}{|c|}{ Munsell colour } & \multirow{2}{*}{$\begin{array}{l}\text { Soil material/textural } \\
\text { class*** }\end{array}$} & \multicolumn{2}{|c|}{ Soil classification } \\
\hline & & & & & $\mathrm{H}_{2} \mathrm{O}$ & $\mathrm{KCl}$ & dry & moist & & PSC (1989) & $\operatorname{PSC}(2011)^{* * * *}$ \\
\hline
\end{tabular}

\section{Gązwa gyttja land}

Profile 1. PSC (2019): gleba gytiowa murszowa (odwodniona) / murshic gyttja soil (drained) WRB (2015): Hypereutric Rheic Murshic Histosol (Limnic)

\begin{tabular}{lllllllllll}
\hline $0-28$ & Md & 20 & 84.48 & 0.0 & 6.99 & 6.86 & $2.5 Y 3.5 / 2$ & $2.5 Y 2 / 2$ & gyttja mursh \\
28-150 & Lc & 21 & 82.72 & 0.0 & 6.80 & 6.67 & $5 Y 3 / 2$ & 5Y 2/2 & $\begin{array}{l}\text { algal gyttja } \\
\text { limni-murshic soil }\end{array}$ \\
\hline
\end{tabular}

Profile 2. PSC (2019): gleba gytiowa murszowa (odwodniona) / murshic gyttja soil (drained) WRB (2015): Hypereutric Rheic Murshic Histosol (Limnic)

\begin{tabular}{|c|c|c|c|c|c|c|c|c|c|c|c|}
\hline $0-27$ & $\mathrm{Md}$ & 22 & 20.56 & 0.0 & 6.14 & 5.87 & $2.5 Y 4 / 1$ & $2.5 Y 2 / 1$ & gyttja mursh & gytiowo- & organiczna \\
\hline $27-160$ & Lc1 & 23 & 87.75 & 0.0 & 6.51 & 6.09 & $5 Y 3 / 2$ & $5 Y 2 / 2$ & algal gyttja & -murszowa/ & -limnowo- \\
\hline $160-245$ & Lc2 & 24 & 67.09 & 0.0 & 6.87 & 6.37 & $5 Y 2 / 1$ & $5 Y 2 / 2$ & coarse detrital gyttja & gyttja-muck soil & murszowa / limni- \\
\hline $245-325$ & Gca & 25 & 1.08 & 9.20 & 8.13 & 7.76 & $5 Y 4 / 2$ & $5 Y 3 / 2$ & $\begin{array}{l}\text { clay gyttja (fluvic } \\
\text { material) }\end{array}$ & & murshic soil \\
\hline
\end{tabular}

Profile 3. PSC (2019): gleba murszowa płytka (węglanowa, odwodniona) / thin murshic soil (calcareous, drained) WRB (2015): Calcic Gleysol (Katoarenic, Drainic, Hyperhumic, Epilimnic)

\begin{tabular}{|c|c|c|c|c|c|c|c|c|c|c|c|}
\hline 0-18 & Md & 26 & 20.94 & 0.27 & 6.91 & 6.45 & 10YR 3/1 & 10YR 2/1 & gyttja mursh & gytiowo- & organiczna \\
\hline $18-42$ & MLc & 27 & 49.00 & 0.53 & 7.15 & 6.80 & $2.5 Y 4 / 2$ & $5 Y 2 / 2$ & murshing fine detrital & -murszowa & limnowo- \\
\hline $42-150$ & Gca & & 8.93 & 25.76 & 7.96 & 7.36 & $5 Y 6 / 3$ & $5 Y 4 / 3$ & $\begin{array}{l}\text { gyttja } \\
\text { sandy loam/gp }\end{array}$ & $\begin{array}{l}\text { /gyttja-muck } \\
\text { soil }\end{array}$ & $\begin{array}{l}\text {-murszowa / } \\
\text { limni-murshic soil }\end{array}$ \\
\hline
\end{tabular}

Profile 4. PSC (2019): gleba gruntowo-glejowa murszowata (limnowęglanowa) / semimurshic gleysol (limni-calcareous) WRB (2015): Eutric Gleysol (Colluvic, Drainic, Humic, Limnic, Nechic)

\begin{tabular}{|c|c|c|c|c|c|c|c|c|c|c|c|}
\hline $0-25$ & Aud & & 13.76 & 0.0 & 6.81 & 6.31 & 10YR $4.5 / 2$ & 10YR 3/1 & semimurshic & murszowata & murszowata / \\
\hline $25-48$ & Lcg & 28 & 58.95 & 0.48 & 7.07 & 6.87 & $2.5 Y 4 / 2$ & $2.5 Y 2 / 1$ & fine detrital gyttja & właściwa / & semimurshic soil \\
\hline $48-150$ & $\mathrm{Lm}$ & 29 & 2.61 & 38.60 & 7.93 & 7.58 & $5 Y 6 / 2$ & $5 Y 5 / 2$ & lacustrine marl & $\begin{array}{l}\text { proper mucky } \\
\text { soil }\end{array}$ & \\
\hline
\end{tabular}

Profile 5. PSC (2019): gleba gytiowa murszowa (limnowęglanowa, odwodniona) / murshic gyttja soil (limni-calcareous, drained) WRB (2015): Calcaric Gleysol (Drainic, Hyperhumic, Limnic)

\begin{tabular}{|c|c|c|c|c|c|c|c|c|c|c|c|}
\hline $0-18$ & $\mathrm{Md}$ & 30 & 32.94 & 0.82 & 6.59 & 6.54 & $2.5 Y 3 / 1.5$ & $2.5 Y 2 / 1$ & gyttja mursh & gytiowo- & organiczna \\
\hline $18-80$ & Lcca & 31 & 23.08 & 66.30 & 7.86 & 7.55 & $5 Y 6 / 2$ & $5 Y 5 / 2$ & proper calcareous gyttja & -murszowa/ & limnowo- \\
\hline $80-150$ & Lm & 32 & 8.25 & 35.12 & 7.54 & 7.23 & $5 Y 6 / 3$ & $5 Y 3 / 2$ & lacustrine marl & gyttja-muck soil & $\begin{array}{l}\text {-murszowa / } \\
\text { limni-murshic soil }\end{array}$ \\
\hline
\end{tabular}

Profile 6. PSC (2019): gleba gytiowa murszowa (limnowęglanowa, odwodniona) / murshic gyttja soil (limni-calcareous, drained) WRB (2015): Calcaric Gleysol (Drainic, Hyperhumic, Limnic)

\begin{tabular}{|c|c|c|c|c|c|c|c|c|c|c|c|}
\hline $0-22$ & $\mathrm{Md}$ & 33 & 31.49 & 2.84 & 7.35 & 7.00 & 10YR 3/1 & $2.5 Y 2 / 1$ & gyttja mursh & gytiowo- & organiczna \\
\hline $22-60$ & Lcca & 34 & 20.13 & 67.46 & 7.90 & 7.56 & $5 Y 7 / 1$ & $5 Y 5 / 2$ & proper calcareous gyttja & -murszowa/ & limnowo- \\
\hline $60-150$ & $\mathrm{Lm}$ & 35 & 7.65 & 33.01 & 7.58 & 7.34 & $5 Y$ 6/2 & $5 Y 4 / 2$ & lacustrine marl & gyttja-muck soil & $\begin{array}{l}\text {-murszowa / } \\
\text { limni-murshic soil }\end{array}$ \\
\hline
\end{tabular}

Profile 7. PSC (2019): gleba murszasta (węglanowa) / postmurshic soil (calcareous) WRB (2015): Gleyic Phaeozem (Pantoarenic, Nechic)

\begin{tabular}{llllllllll}
\hline $0-32$ & Aud & 3.44 & 0.0 & 5.56 & 4.58 & 10YR 5/2 & 10YR 3/2 & postmurshic/pl & murszasta / \\
$32-52$ & ACca & 1.95 & 2.42 & 7.04 & 6.96 & 10YR 5/3 & 10YR 4/3 & loamy sand/pg \\
$52-67$ & Cca & 0.84 & 12.35 & 8.45 & 7.61 & 10YR 6/2 & 10YR 4.5/3 & sand/ps \\
$67-88$ & Ccagg1 & 0.28 & 8.21 & 8.93 & 7.98 & $2.5 Y$ 6/2 & $2.5 Y$ 4/3 & sand/pl \\
$88-150$ & Ccagg2 & 0.23 & 5.66 & 8.66 & 8.02 & $2.5 Y$ 6/3 & $2.5 Y 4 / 3$ & sand/pl \\
\hline
\end{tabular}


Table 1, continue

\begin{tabular}{|c|c|c|c|c|c|c|c|c|c|c|}
\hline \multirow{2}{*}{$\begin{array}{l}\text { Depth } \\
{[\mathrm{cm}]}\end{array}$} & \multirow{2}{*}{$\begin{array}{l}\text { Soil } \\
\text { horizon* }\end{array}$} & \multirow{2}{*}{$\begin{array}{l}\text { Bottom } \\
\text { lake } \\
\text { deposit } \\
\text { No.** }\end{array}$} & \multirow{2}{*}{$\begin{array}{l}\text { LOI } \\
{[\%]}\end{array}$} & \multirow{2}{*}{$\begin{array}{l}\mathrm{CaCO}_{3} \\
{[\%]}\end{array}$} & \multicolumn{2}{|l|}{$\mathrm{pH}$} & Munsell colour & \multirow{2}{*}{$\begin{array}{l}\text { Soil material/textural } \\
\text { class*** }\end{array}$} & \multicolumn{2}{|c|}{ Soil classification } \\
\hline & & & & & $\mathrm{H}_{2} \mathrm{O}$ & $\mathrm{KCl}$ & moist & & PSC (1989) & $\operatorname{PSC}(2011)^{* * * *}$ \\
\hline
\end{tabular}

Profile 8. PSC (2019): gleba namurszowa gytiowa (płytka, węglanowa) / earth-covered murshic gyttja soil (shallow, calcareous) WRB (2015): Dystric Gleysol (Endoloamic, Drainic, Limnic, Nechic)

\begin{tabular}{|c|c|c|c|c|c|c|c|c|c|c|c|}
\hline $0-11$ & Aud & & 16.22 & 0.0 & 4.00 & 3.33 & 10YR $4.5 / 2$ & 10YR $2 / 2$ & semimurshic/pg & namurszowa / & murszowata / \\
\hline $11-50$ & MLc & 36 & 44.49 & 0.0 & 4.62 & 4.05 & $5 Y 4 / 2$ & $5 Y 2 / 1$ & murshing fine detrital & mucky soil & semimurshic soil \\
\hline 50-150 & Gca & & 2.35 & 4.25 & 7.93 & 7.21 & $2.5 Y 6 / 3$ & $2.5 Y 4 / 3$ & $\begin{array}{l}\text { gyttja } \\
\text { sandy loam/gp }\end{array}$ & & \\
\hline
\end{tabular}

Profile 9. PSC (2019): gleba murszowa płytka (odwodniona) / thin murshic soil (drained) WRB (2015): Dystric Rheic Murshic Histosol (Katoarenic, Epilimnic)

\begin{tabular}{|c|c|c|c|c|c|c|c|c|c|c|c|}
\hline $0-27$ & $\mathrm{Md}$ & 37 & 67.00 & 0.0 & 4.63 & 4,24 & $2.5 Y 4 / 1$ & $2.5 Y 2 / 1$ & gyttja mursh & gytiowo- & organiczna \\
\hline $27-45$ & MLc & 38 & 62.11 & 0.0 & 5.39 & 4.80 & $2.5 Y 3 / 2$ & $2.5 \mathrm{Y} 2 / 1$ & murshing coarse detrital & -murszowa / & limnowo- \\
\hline $45-150$ & G & & 1.00 & 0.13 & 6.99 & 6.07 & $2.5 Y 6 / 2$ & $2.5 Y 4 / 2$ & $\begin{array}{l}\text { gyttja } \\
\text { loamy sand/pg }\end{array}$ & gyttja-muck soil & $\begin{array}{l}\text {-murszowa / } \\
\text { limni-murshic soil }\end{array}$ \\
\hline
\end{tabular}

Łąki Dymerskie gyttja land

Profile 1. PSC (2019): regosol typowy (węglanowy) / typical regosol (calcareous) WRB (2015): Eutric Regosol (Pantoarenic, Humic)

\begin{tabular}{|c|c|c|c|c|c|c|c|c|c|}
\hline $0-28$ & Ap & 2.95 & 0.51 & 7.41 & 6.86 & 10YR $4.5 / 3$ & 10YR 3/2 & humous sand/pl & arenosol / \\
\hline $28-65$ & Cca1 & 0.69 & 0.27 & 7.58 & 7.06 & 10YR 5/4 & 10YR 4/4 & sand/pl & właściwy / \\
\hline $65-150$ & Cca2 & 0.50 & 10.49 & 8.84 & 8.01 & 10YR 7/2 & 10YR 6/2 & sand/pl & proper arenosol \\
\hline
\end{tabular}

Profile 2. PSC (2019): gleba murszasta (słabo gruntowo-glejowa) / postmurshic soil (weakly gleyic) WRB (2015): Mollic Gleysol (Pantoarenic, Colluvic, Drainic, Humic, Nechic)

\begin{tabular}{|c|c|c|c|c|c|c|c|c|c|c|}
\hline $0-38$ & Aup & 6.18 & 0.0 & 6.13 & 5.56 & 10YR 4/1 & 10YR 2.5/1 & postmurshic/pl & murszasta/ & murszasta \\
\hline $38-57$ & $\mathrm{AuC}$ & 3.67 & 0.0 & 5.81 & 4.77 & 10YR 5/2 & 10YR 3/1.5 & postmurshic /pl & muckous soil & typowa / typical \\
\hline $57-150$ & Cgg & 0.34 & 0.45 & 7.08 & 6.87 & $2.5 Y 6 / 3$ & $2.5 Y 4 / 3$ & sand/pl & & postmurshic soil \\
\hline
\end{tabular}

Profile 3. PSC (2019): gleba murszasta (węglanowa, słabo gruntowo-glejowa, nasypowa) / postmurshic soil (calcareous, weakly gleyic, heaped) WRB (2015): Eutric Gleysol (Endoarenic, Drainic, Humic, Limnic, Nechic)

\begin{tabular}{|c|c|c|c|c|c|c|c|c|c|c|c|}
\hline $0-31$ & Aup & & 6.21 & 1.49 & 7.39 & 7.04 & 10YR 4/1 & 10YR 3/2 & postmurshic/pl & murszasta/ & murszasta \\
\hline $31-51$ & $\mathrm{Lc}$ & 39 & 45.12 & 0.0 & 4.84 & 4.33 & $2.5 Y 4 / 2$ & $2.5 Y 2 / 1$ & fine detrital gyttja & muckous soil & typowa / typical \\
\hline $51-150$ & Ccagg & & 0.55 & 5.73 & 8.37 & 7.78 & $2.5 Y$ 6/2 & $2.5 Y 5 / 3$ & sand/pl & & postmurshic soil \\
\hline
\end{tabular}

Profile 4. PSC (2019): gleba murszasta (węglanowa, nasypowa, pogytiowa) / postmurshic soil (calcareous, heaped, postgyttja) WRB (2015): Hypereutric Rheic Drainic Histosol (Limnic, Nechic, Areninovic)

\begin{tabular}{|c|c|c|c|c|c|c|c|c|c|c|c|}
\hline $0-22$ & Aup & & 11.10 & 0.00 & 6.46 & 6.11 & 10YR 3/2 & 10YR 2/1 & semimurshic/pl & murszowata & organiczna \\
\hline $22-37$ & Auca & & 3.14 & 4.39 & 7.55 & 7.33 & 10YR 5/2 & 10YR 4/1 & postmurshic/pl & właściwa/ & limnowo- \\
\hline $37-44$ & Lc1 & 40 & 52.98 & 0.0 & 6.07 & 5.81 & $2.5 Y 4 / 1$ & $2.5 Y 2 / 1$ & fine detrital gyttja & proper mucky & -murszowa / \\
\hline $44-150$ & Lc2 & 41 & 61.19 & 0.0 & 6.01 & 5.80 & $2.5 Y 3 / 1$ & $2.5 Y 2 / 1$ & coarse detrital gyttja & soil & limni-murshic soil \\
\hline
\end{tabular}

Profile 5. PSC (2019): regosol typowy (węglanowy) / typical regosol (calcareous) WRB (2015): Eutric Regosol (Pantoarenic)

\begin{tabular}{|c|c|c|c|c|c|c|c|c|c|c|}
\hline $0-24$ & Ap & 1.97 & 1.28 & 8.06 & 7.45 & 10YR 5/3 & 10YR 3.5/2 & humous sand/pl & arenosol & arenosol / \\
\hline $24-51$ & Cca1 & 0.29 & 8.19 & 8.96 & 8.00 & 10YR 7/2 & 10YR $6 / 2$ & sand/pl & właściwy / & arenosol \\
\hline $51-69$ & Cca2 & 0.32 & 10.64 & 8.89 & 8.01 & 10YR $7 / 3$ & 10YR 6/3 & sand/pl & proper arenosol & \\
\hline $69-88$ & Cca3 & 0.19 & 6.03 & 8.96 & 8.05 & 10YR 7.5/2 & 10YR 6/3 & sand/pl & & \\
\hline $88-150$ & Cca4 & 0.25 & 8.44 & 8.92 & 8.12 & 10YR 8/2 & 10YR 6.5/3 & sand/pl & & \\
\hline
\end{tabular}

Profile 6. PSC (2019): regosol typowy (węglanowy) / typical regosol (calcareous) WRB (2015): Eutric Regosol (Pantoarenic, Humic, Nechic)

\begin{tabular}{|c|c|c|c|c|c|c|c|c|c|c|}
\hline $0-24$ & Aup & 4.46 & 0.45 & 7.17 & 6.78 & 10YR 5/2 & 10YR 2/2 & postmurshic/pl & murszasta/ & murszasta \\
\hline $24-45$ & Cca1 & 1.22 & 0.82 & 8.12 & 7.60 & 10YR 5/4 & 10YR 3/4 & sand/pl & muckous soil & typowa/ typical \\
\hline $45-150$ & Cca2 & 0.32 & 4.79 & 8.65 & 8.04 & 10YR $7 / 3$ & 10YR 5/3 & sand/pl & & postmurshic soil \\
\hline
\end{tabular}


Table 1, continue

\begin{tabular}{|c|c|c|c|c|c|c|c|c|c|c|}
\hline \multirow{2}{*}{$\begin{array}{l}\text { Depth } \\
\text { [cm] }\end{array}$} & \multirow{2}{*}{$\begin{array}{l}\text { Soil } \\
\text { horizon* }\end{array}$} & \multirow{2}{*}{$\begin{array}{l}\text { Bottom } \\
\text { lake } \\
\text { deposit } \\
\text { No.** }\end{array}$} & \multirow{2}{*}{$\begin{array}{l}\text { LOI } \\
{[\%]}\end{array}$} & \multirow{2}{*}{$\begin{array}{l}\mathrm{CaCO}_{3} \\
{[\%]}\end{array}$} & \multicolumn{2}{|l|}{$\mathrm{pH}$} & Munsell colour & \multirow{2}{*}{$\begin{array}{l}\text { Soil material/textural } \\
\text { class*** }\end{array}$} & \multicolumn{2}{|c|}{ Soil classification } \\
\hline & & & & & $\mathrm{H}_{2} \mathrm{O}$ & $\mathrm{KCl}$ & moist & & PSC (1989) & $\operatorname{PSC}(2011)^{* * * *}$ \\
\hline
\end{tabular}

Profile 7. PSC (2019): gleba murszowata typowa (nasypowa) / typical semimurshic soil (heaped) WRB (2015): Mollic Gleysol (Endoarenic, Drainic, Humic, Limnic, Nechic, Areninovic)

\begin{tabular}{|c|c|c|c|c|c|c|c|c|c|c|c|}
\hline $0-39$ & Aud & & 11.10 & 0.0 & 6.31 & 6.21 & 10YR $4.5 / 2$ & 10YR 2/1 & semimurshic/pl & murszowata & murszowata / \\
\hline $39-61$ & MLc & 42 & 22.01 & 0.0 & 6.01 & 5.76 & 10YR 3/2 & $2.5 Y 2 / 1$ & murshing gyttja & właściwa/ & semimurshic soil \\
\hline $61-76$ & Cgg1 & & 4.95 & 0.0 & 6.43 & 6.17 & $2.5 Y 5 / 2$ & $2.5 Y 3 / 2$ & sand with humus & proper mucky & \\
\hline $76-115$ & Cgg2 & & 8.57 & 0.0 & 5.84 & 5.74 & $2.5 Y 3 / 2$ & $2.5 Y 2 / 1$ & admixture/pl & soil & \\
\hline $115-150$ & Cgg3 & & 1.13 & 0.0 & 6.44 & 6.38 & $2.5 Y 6 / 2$ & $2.5 Y 4 / 2$ & $\begin{array}{l}\text { sand with humus } \\
\text { admixture/pl } \\
\text { sand/pl }\end{array}$ & & \\
\hline
\end{tabular}

Profile 8. PSC (2019): gleba gytiowa (odwodniona) / gyttja soil (drained) WRB (2015): Dystric Rheic Drainic Sapric Histosol (Limnic)

\begin{tabular}{llllllllll}
\hline 0-29 & Lc1 & 43 & 40.91 & 0.0 & 5.53 & 5.44 & $2.5 Y 3 / 2$ & $2.5 Y 2 / 1$ & fine detrital gyttja \\
$29-150$ & Lc2 & 44 & 40.76 & 0.0 & 5.31 & 5.24 & $2.5 Y 3 / 1$ & $2.5 Y 2 / 1$ & fine detrital gyttja
\end{tabular}

LOI - loss-on-ignition

* - according to Polish Soils Classification (2019)

** - as presented on triangle, Fig. 3

*** - according to the Soil Texture Classification of Soil Science Society of Poland (2009)

**** - English equivalents for the taxa names according to Świtoniak et al. (2016)

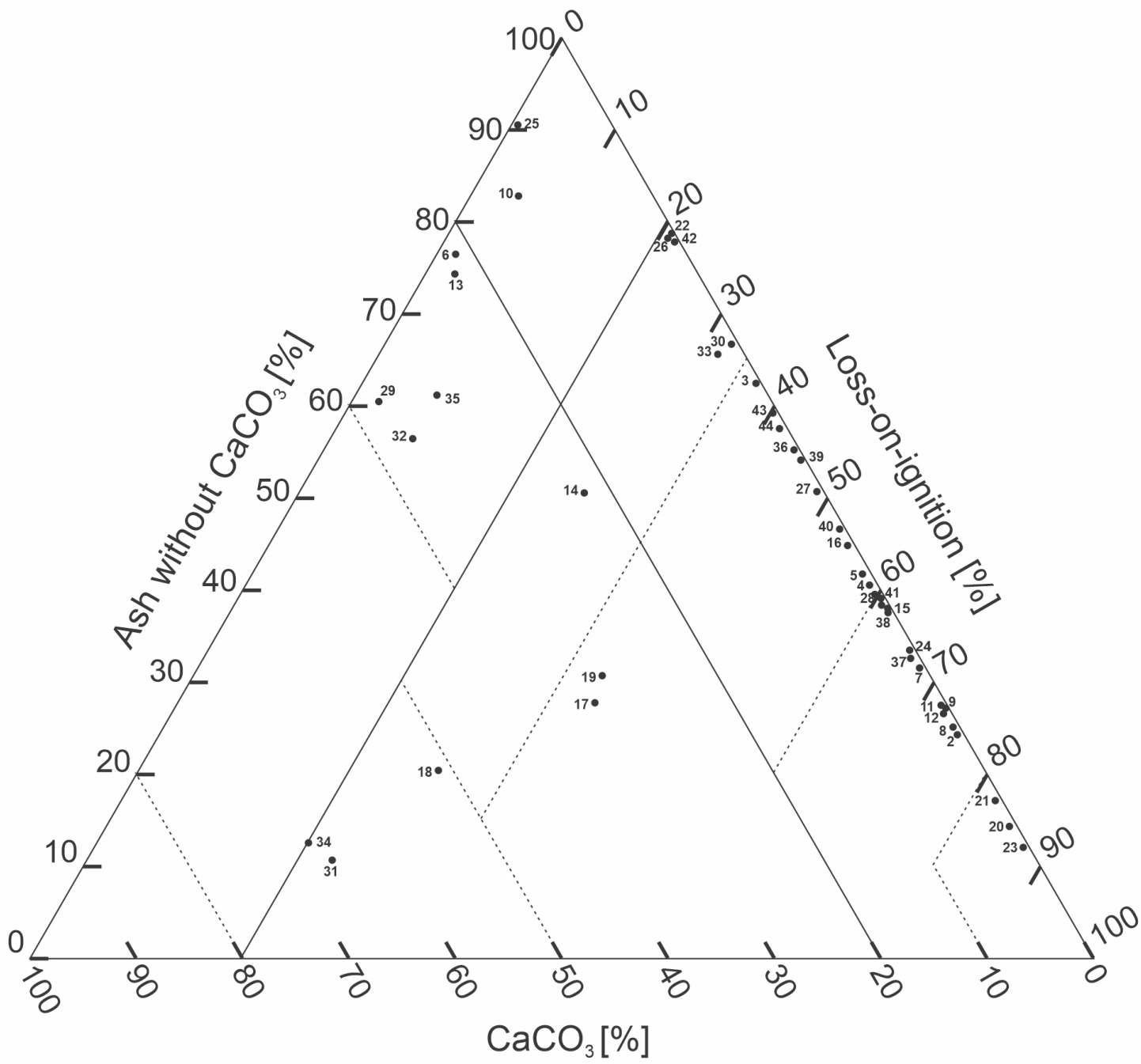

Fig. 3. Classification of samples of bottom lake deposits of studied soils according to PSC (2019) 
(gyttja mursh), 4 samples represented transition horizons (MLcmurshing gyttja), while the remaining 23 samples belong to various types of limnic materials untransformed by aerobic pedogenetic processes. When organic gyttjas are affected by murshing, distinct layering of the surface horizon appears. Its upper part overgrown with plant roots (the turf sub-layer) attains the crumby or fine prismatic structure, which deeper into the soil it turns into the structure referred to as the laminar one. It consists of aggregates $2-3 \mathrm{~cm}$ in diameter and 1-3 $\mathrm{mm}$ in thickness. With depth, aggregates are becoming larger and the structure can be termed as the coarse prismatic one. Gradually, beside horizontal layers, there emerge vertical crevices and fissures reaching down to the groundwater oscillation level. The development of characteristic sublayers in the soil horizon affected by the murshing process depends on several factors, including the depth of a gyttja deposit, duration and intensity of drainage, level of groundwater and plant cover (Uggla, 1962, 1967; Chmieleski and Zeitz, 2006). It was observed in the analysed soil profiles that when the soil surface was covered with a mineral overlayer (of natural or anthropogenic origin), the murshing process deep into the soil profile decelerated. Similar conclusions are drawn in a paper describing changes of lake catchment in Brodnickie Lakeland (Mendyk et al., 2016).

Noteworthy is the fact that two samples were classified as lacustrine clay (clay gyttja) (Fig. 3), which according to Markowski (1980) is found rather rarely and usually in the lower parts (floor) of the deposit. However, Uggla (1969a) mentioned that clay gyttja and clay-detrital gyttja can also be found in the upper layers of deposits, where it serves as the parent material of soils. Clay gyttja is genetically connected with the early stages of the development of a lake, when plankton production is low and therefore these organisms contribute less to accumulated sediments. When clay gyttja runs off or crawls down the slope, it most often accumulates in the lowest parts of the lake basin, where it forms thick layers of deposits (Uggla, 1971). In the analysed soil profiles, clay gyttja was found in deeper layers (108-180 cm) in profile 4 (Łąki Staświńskie) and (245-325 cm) in profile 2 (Gązwa) (Table 1).

\subsection{Classification of soils}

As regards the taxonomy of soil profiles composed of limnic materials with greater thickness, all compared soil classifications are quite consistent. These soils, if not undergoing the murshing process, are classified in PSC6 as gyttja soils (Polish - gleby gytiowe). Profiles of such soils were identified in the middle parts (further from the shores) of drained lakes (profile 5 in Łąki Staświńskie, profile 8 in Łąki Dymerskie). In the upper, surface layers of the profile, these gyttjas do not display the plate (laminate) structure, characteristic for gyttja murshes, but they are pervaded by roots of reeds and sedges. Some subtle symptoms of pedogenesis are associated with the time period immediately after the discharge of water of lakes, when these materials were exposed to the influences of atmospheric factors, which is indicated by their darker colour (Uggla, 1969b). After World War Two, the level of water increased as a result of the so-called secondary bogging (re-swamping). At present, the level of water is on par with the surface of land, and soils are covered with reed plant communities, which are now demonstrating the peatforming abilities. In consequence, a layer of reed peat accumulates on the superficial layer of gyttja in the form of plant roots turned into peat. These profiles (no 5 and 8) were classified as gyttja soils in PSC (1989) and as typical limnic soils in PSC (2011). According to WRB (2015), they were classified as Hypereutric Rheic Drainic Sapric Histosol (Limnic) and Dystric Rheic Drainic Sapric Histosol (Limnic), respectively.

When analysed gyttja soils undergo the murshing process, they are classified according to PSC6 (2019) as gyttja murshic soils (drained) (Polish - gleba murszowa gytiowa) (profiles no 3, 4 in Łąki Staświńskie), or as murshic gyttja soils (Polish - gleba gytiowa murszowa) (profiles no 8 in Łąki Staświńskie, and 1, 2, 5, 6 in Gązwa gyttja land). As the thickness of the murshic layer in profiles 3 and 4 in Łąki Staświńskie exceeds $30 \mathrm{~cm}$, they were classified as gyttja murshic soils (PSC6). The classification of profile 8 in Łąki Staświńskie and profiles 1, 2, 5, 6 in Gązwa gyttja land issues from the murshic layer being less than $30 \mathrm{~cm}$ thick. According to WRB (2015), all these profiles were classified as Murshic Histosols (Limnic), except for profiles 5 and 6 in Gązwa, which - because of its thickness of the organic horizon less than $40 \mathrm{~cm}$ - were classified as Calcaric Gleysols. Both earlier versions of PSC $(1989,2011)$ classified these profiles as gyttja-muck soils or limni-murshic soils, respectively.

Profiles 3 and 4 from the Łąki Staświńskie gyttja land were found to contain thin layers of sand (Table 1). Such a layer in profile 3 occurs at a depth of 76-81 $\mathrm{cm}$, whereas in profile 4 it lies deeper, at 100-108 cm. These sand layers separate the overlying detrital gyttja from the underlying lacustrine marl (profile 3) and clay gyttja layer (profile 4). Because the sand layer separates the limnic sediments with totally different chemical composition, as the layer underneath consists of mineral deposits while the upper ones are organic gyttjas, it can be suspected that they also differ in age. Because this finding concerns the profiles located near the shore of a former lake, it can be assumed that the sandy layer was accumulated by colluvial and/or eolic processes when the lake shores had been exposed, deforested for agricultural use of the land in the lake catchment or left barren due to a forest fire. A similar interpretation of the genesis of sandy strata in profiles of gyttja soils was given by Uggla and Mirowski (1968) (cf. Figure 1 given in their paper) and by Uggla (1964, 1971).

Three profiles were classified according to PSC6 as thin murshic soils (Polish - gleby murszowe płytkie) (profile no 2 in Łąki Staświńskie and profiles nos 3 and 9 in Gązwa). They are characterised by a superficial murshic layer of the thickness of more than $30 \mathrm{~cm}$, and the total thickness of organic matter is $\leq 50 \mathrm{~cm}$. All these profiles are located nearer the edge of the former lake. In the profiles found in Gązwa gyttja land, there is a transition layer (MLc) underneath the murshic layer (Md) where gyttja mursh is gradually transformed to detrital gyttja. According to WRB (2015), these profiles were classified as Eutric Gleysol, Calcic Gleysol, and Murshic Histosol, respectively.

Soils developed from bottom lake deposits rich in calcium carbonate, which Uggla $(1971,1976)$ suggested to name post-lacustrine or Quaternary rendzinas, are an interesting type of soils. 
They have been recently studied by Lemkowska and Sowiński (2018) in the Masurian Lakeland and by Meller (2006) and Jarnuszewski and Meller $(2018,2019)$ in Western Pomerania, in Poland. Although a certain amount of calcium carbonate was often presented in the analysed limnic materials (Fig. 3), cases when it exceeds $20 \%$, which would justify their classification as carbonate gyttjas, are rather infrequent. Six samples were identified as calcareous gyttjas while five other samples were determined to belong to the meadow limestone (marl) class. None of the profiles was classified as representing limnic ordinary rendzina (Polish - rędzina właściwa, podtyp pojeziorna) according to the criteria of soil classification laid out in PSC6. However, this classification system allows the identification of soils rich in carbonates at the level of varieties. The following varieties were applied to the above profiles limni-calcareous, calcareous, and deep calcareous (Table 1).

Greater typological diversity appears among soils developed on edges of drained lakes. This is the consequence of numerous, overlapping processes affecting the shallow layers of limnic materials, which were covered with thin layers of peat or peat-like materials to be then modified by the admixture of colluvial materials, and possibly also eolic ones. Some effect is also produced by the gleying process (at present, after dewatering, most often in the upper layer identified as a relict one). As these soils lie higher than the current groundwater level, the soil material after draining underwent intensive transformation and was homogenised due to mechanical tillage (ploughing).

The lakeshore area in Łąki Staświńskie was found to contain the soils (profiles nos 6 and 7) classified according to PSC6 as typical gleysol (calcareous) and murshic gleysol (limni-calcareous), respectively. Profile 7 comprises a thin layer (mixed layer Aca/Lm) composed of sand with gyttja admixture, and the entire profile was determined to have a high content of calcium carbonate, which was reflected in the classification of this soil according to WRB (2015): Calcaric Histic Gleysol (Katoloamic, Drainic, Limnic).

Profile 4 in Gązwa gyttja land has a superficial, 25-cm thick layer (Aud) of semimurshic material. It is underlain by a $25-48-\mathrm{cm}$ thick layer of fine detrital gyttja, and lacustrine marl underneath. In line with PSC6, this profile was classified as semimurshic gleysol (limni-calcareous) (Polish - gleba gruntowo-glejowa murszowata (limnoweglanowa). The location of this profile relative to the slope implicates the colluvial genesis of the superficial layer, which was reflected in the taxonomic position of this soil according to WRB (2015): Eutric Gleysol (Colluvic, Drainic, Humic, Limnic, Nechic). Similarly, profile 8 from the same gyttja land contained in its topmost layer (Aud) a thin, 11-cm thick layer of semimurshic material, underlain by murshing fine detrital gyttja (11-50 cm), with sandy loam below. The location of the profile near the shore of the former lake and the steep slope in the nearest proximity suggest the colluvial origin of the soil material in the top layer of this profile. According to PSC6, this profile represents earth-covered murshic gyttja soil (shallow, calcareous) (Polish - gleba namurszowa gytiowa (płytka, weglanowa).

The soils surrounding the gyttja lands, profile 7 (Gązwa) and profile 2 (Łąki Dymerskie) were classified as postmurshic soils (Polish - gleby murszaste), which was justified by the thick- ness of the arenimurszik horizon (over $30 \mathrm{~cm}$ ), which gradually transforms into the transition horizon (ACca or AuC), which is characteristic feature of this type of soils (Łabaz and Kabała, 2016; PSC6). In the analysed soils, the arenimurszik horizon had developed as a result of concurrent soil forming processes and slope processes. The colluvial process overlapped with the in situ accumulation of peaty organic matter in the lakeshore of the former lakes that was vigorously mixed with sand. Due to pedogenic transformation of the surface horizon, the soil largely lost the layering typical of deposits accumulating on slopes. The location in the near vicinity of a slope, however, explains the genesis of the layer identified now as arenimurszik. Considering the more strongly manifested effect of colluvial processes, it was decided to classify profile 2 in Łąki Dymerskie gyttja land as Mollic Gleysol (Pantoarenic, Colluvic, Drainic, Humic, Nechic) according to WRB (2015).

In Łąki Dymerskie gyttja land, the lake sediments were covered with a layer of sand transported here by man (the Rimpau method). As the water level declined considerably, the deposited materials underwent the pedogenic transformation. The surface materials, created as a result of mixing with sand and depending on their content of organic matter (LOI), are classified as murshic ( $\geq 20 \%$ ), semimurshic (10-20\%) and postmurshic soil formations (3-10\%) in all the three PSC versions (1989, 2011, 2019).

Profiles 3 and 4 from Łąki Dymerskie, according to PSC6, were classified as postmurshic soils (Polish - gleba murszas$t a$ ), and profile no 7 as typical semimurshic soil (Polish - gleba murszowata typowa). The PSC6 enabled the classification of these soils to the level of variety. Based on the content of calcium carbonate in profiles 3 and 4 ( $\geq 2 \%$ ), their name was supplemented by adding the variety - calcareous (węglanowa). In profile 3, under the Aup horizon, there is a thin, 20-cm-thick layer of fine detrital gyttja, and in profile 4 (further from the shore of the former lake), a layer of detrital gyttja was found at a depth of $37-150 \mathrm{~cm}$. In the case of profile no 4 the variety was added postgyttja (Polish - pogytiowa). The surface postmurshic layers (profiles 3 and 4) as well as the semimurshic layer (profile no 7) had developed from loose sand transported by man from the islands emerged after dewatering and from the area surrounding the gyttja land. The sand excavation pits are still visible in the nearest proximity of the gyttja land, and there is a written report on conducting the above land improvement measure, published to convince users of such areas about benefits of sanding and fertilising gyttja land. Water from Dymer Lake was discharged in 1871-1876 and the emerging bottom sediments were turfed with grass mixtures by sowing grass seeds directly on the ice cover. In 1885, people began to cover the meadows with a 6- or 11-centimeter-thick layer of sand, after which mineral fertilisation with Thomas slag (basic phosphate) and kainite (which was an unusual practice on grasslands in the then East Prussia) and a mixture of grasses was re-sown (Körnig, 1908). E. Arend, the administrator of state-owned Łąki Dymerskie gyttja land, carried out a planned experiment concerning the effect of the thickness of a sand layer and mineral fertilisation (Thomas slag, potassium salt) on the yielding of meadows on gyttja soils (Arend, 1894). The thickness of the lay- 
ers of sand spread over Łąki Dymerskie gyttja land was $6 \mathrm{~cm}$ (5.23 ha), $8 \mathrm{~cm}$ (25.58 ha) and 10-11 cm (101.79 ha). Thus, over half of the gyttja land underwent this treatment. Arend (1894) gives 21 examples of gyttja and peat lands in different parts of the Mazury region where layers of sand from $1 \mathrm{~cm}$ to $15 \mathrm{~cm}$ thick were applied in addition to kainite and Thomas slag, but also with manure, slurry and compost fertilisation. The cases he provided prove that this was a popular treatment in all East Prussia and covered (end of 19th century) at least a few hundred hectares of meadows.

It is worth mentioning here that the sand cover cultivation (sand-mantled culture) applied in Łaki Dymerskie was referred to as Sanddeckkultur in the German language and can be seen as a certain modification of the method developed by Theodor Rimpau (1822-1888). The modification consisted in spreading a layer of sand on lacustrine bottom sediments soon after they emerged rather than on the surface of low peatland. Another change was that the sand was excavated from nearby hills and not from the bottom of a drained lake. In its classical form, the Rimpau bog sand-cover cultivation leads to the formation of a characteristic landscape with narrow grassland areas which are separated from each other at regular intervals by ditches, which were created by excavating sand for land amelioration (Sauerbrey et al., 2003). Sanding peat soils is an expensive and time-consuming treatment as it requires the application of 1,800-2,200 t/ha of sand, but due to the high returns it was popular in Germany and adjacent countries at the turn of 19th and 20th century (Ermert, 1963). The large share of organic soils with a sandy top layer in the present soil cover in Germany has been emphasised by Frank et al. (2017) and Säurich et al. (2019).

The excavated sand used to ameliorate Łąki Dymerskie gyttja land, contained considerable amounts of calcium carbonate, especially collected from deeper layers, hence the postmurshic layers (Aup) developed with its contribution contain more $\mathrm{CaCO}_{3}$ than the underlying soil layers (in profile 3, the content of calcium carbonate is $1.49 \%$ but in profile 4 it peaks at $4.39 \%$ ). As there is no doubt here about the anthropogenic origin of the material forming the surface layers, the names of soils in profiles 3 , 4 and 7 in Łąki Dymerskie gyttja land were added the name of the variety, such as heaped (nasypowa). Considering the added layers of sand (6-11 cm thick) and the current thickness of the postmurshic soil layers $(31-39 \mathrm{~cm})$ as well as their content of organic matter, it becomes obvious that they developed due to the intensive mixing of the transported sand (ploughing) with the topmost layers of detrital gyttja, with some minor contribution of colluvial and eolic material. As the beginning of sand introduction is known, it is possible to trace the rate of formation of arenimurszik horizon. In the earlier versions of PSC $(1989,2011)$, these profiles are classified according to the character of surface layer (Table 1). However, according to WRB (2015), they are classified as Gleysols (profile no 3 and 7) and Histosols (profile no 4), which enables a more detailed description of the composition of soil profiles owing to the use of qualifiers, especially supplementary ones, e.g. profiles 3 and 7 were assigned the following qualifiers: Endoarenic, Drainic, Humic, Limnic, Nechic, Areninovic, which reflect their complex composition of profiles and the history of the development of these soils.
The soils around the two gyttja lands were classified according to PSC6 as regosols (profile 1, Łąki Staświńskie, profiles 1, 5 and 6 Łąki Dymerskie). Based on the content of organic matter in the surface layers, profile 1 from Łąki Staświńskie was classified as humic regosol, whereas the remaining profiles were stated to represent the typical regosols. It should be noted that all these profiles belong to the calcareous variety. What is characteristic of these profiles is that the content of calcium carbonate is lower in the surface layers but much greater in the parent material, where in some cases it surpasses $10 \%$. The depletion of the upper layers from calcium carbonate proves that the compound had been washed out. The calcium carbonate leached from these soils was deposited in the basins of nearby lakes, thereby forming layers of calcareous gyttja and lacustrine limestone. The extent to which soils in the Kruklin Lake catchment basin, situated in the Great Masurian Lakes Region, had been leached from calcium was estimated by Gąsiorowski (2001) to be 300-350 kg of calcium carbonate per $1 \mathrm{~m}^{2}$ of the catchment.

One of the differences between PSC6 and its earlier versions is the classification of soils with a thin $(\leq 30 \mathrm{~cm})$ surface layer containing $3-10 \%$ of SOM of the postmurshic character to humic regosols and typical regosols. In the previous PSC versions, they used to be classified as muckous soils (PSC 1989) or typical postmurshic soils (PSC, 2011). This change also pertains to profile 1 from Łąki Staświńskie, where the Aud horizon composed of postmurshic material is $28 \mathrm{~cm}$ thick, and there is a transition horizon CcaA (28-52 cm thick) underneath, which does not satisfy the parameters specified in PSC6 for the arenimurszik horizon. It is characterised by a poorly developed structure, typical of parent material. Despite the relatively high content of LOI (1.53\%), it only contains $0.19 \%$ of $C_{\text {org. }}$ (Eachacz et al., 2009), and fails to meet the criterion of organic carbon content for arenimurszik horizons ( $\geq 0,6 \%$ ) set in PSC6. This horizon of sandy loam texture has substantial content of $\mathrm{CaCO}_{3}(7.38 \%)$ and comprises organic matter in the form of illuvial humus leached from the topmost soil. Profile 6 from Łąki Dymerskie represents a similar case, as the surface Aup horizon is only $24 \mathrm{~cm}$ thick, which does not allow us to classify this soil as postmurshic soil according to PSC6.

The classification of soils developed from bottom sediments of former lakes and those in their immediate surroundings, presented above, implicates that the compared classifications are highly convergent, and the most recent version PSC6 is the continuation and evolution of the earlier versions. However, owing to the consistent application of quantitative parameters for the identification of soil materials and diagnostic horizons as well as the exclusion key, PSC6 enables the user to classify unequivocally all soils to adequate taxonomic units. Thus, the PSC6 differs from the previous editions, which - despite the inclusion of a series of quantitative parameters - retained many features of a typological division, where soil is compared to the predetermined type (model). The subsequent versions of the PSC took into account the secondary transformation of organic soil materials as a result of pedogenesis after the draining of soils, which is the contribution made by Polish scholars to knowledge of organic soils (e.g. Okruszko, 1976; Okruszko and Ilnicki, 2003; Łabaz and Kabała, 2016; Łachacz and Kalisz, 2016). Among the soil materials transformed by pedogenesis, based on the content of organic 
matter and thickness of the soil layer containing thereof, two diagnostic horizons are distinguished: murszik and arenimurszik (PSC6). Uggla (1962) was the first researcher who described the specific nature of mursh-forming process in the case of organic (detrital) gyttja, the phenomenon of soil mass dividing into layers, and the formation of the plate (laminate) structure, and the accompanying processes of swelling and shrinking of gyttja. This specific character has been emphasised by the following researchers exploring drained gyttja lands (e.g. Chmieleski and Zeitz, 2006). Further evolution of mursh under aerobic pedogenesis leads to the depletion of organic matter, its intensive mixing with mineral subsoil, which - when sands are involved - results in the formation of semimurszik and postmurszik soil materials (in Polish - utwór murszowaty, utwór murszasty) (Łabaz and Kabała, 2016), denoted in the PSC6 as the diagnostic horizon called arenimurszik.

\section{Conclusions}

SGP6 allows the user to achieve a more precise classification of analysed soils than the previous editions of this classification system were able to do. One reason is the possibility of using lower taxonomic units, such as a soil variety. This advantage of PSC6 is especially evident when dealing with soils located at edges of former lakes, because their profiles comprise thin layers of different origin and characteristics. This is reflected by the number of subtypes that the soils analysed in this study fell into, namely 12 subtypes in PSC (2019), 7 in PSC (2011) and 8 in PSC (1989). Among the soils studied, most profiles were classified as limnic soils (7), then murshic soils (6), semimurshic soils (5), regosols (4), and gleysols (3). All the analysed soil classifications enable us to identify drained soils with the surface horizons transformed in the course of pedogenesis (murszik, arenimurszik). However, WRB (2015) does not provide soil horizons and materials describing further degrading transformation of mursh soil to soils containing less than $12 \%$ of $\mathrm{C}_{\text {org. }}$ As murshing is a widespread process, WRB should enable the user to identify subsequent stages in the evolution of post-bog soils, for example by implementing appropriate qualifiers. Łąki Dymerskie gyttja land was found to encompass an arenimurszik horizon developed from sand that had been transported there in order to improve gyttja soils. This treatment was a certain modification of the sand-covering of low peatlands (Rimpau method) in the sense that it was applied to gyttja lands and the sand was excavated from nearby hills rather than from ditches dug out in the area submitted to this treatment.

\section{Acknowledgments}

The results presented in this paper were obtained as part of a comprehensive study financed by the University of Warmia and Mazury in Olsztyn, Faculty of Agriculture and Forestry, Department of Soil Science and Microbiology (grant No. 30.610.005110).

\section{References}

Arend, E., 1894. Masuriche Moorwiesen und deren Erträge. Georgine. Eine Zeitschrift für landwirtschaftliche Kultur (Königsberg) 62(43), 398-400; 62(43), 407-408.

Berglund, Ö., Berglund, K., 2010. Distribution and cultivation intensity of agricultural peat and gyttja soils in Sweden and estimation of greenhouse gas emissions from cultivated peat soils. Geoderma 154, 173-180. https://doi.org/10.1016/j.geoderma.2008.11.035

Chmieleski, J., Zeitz, J., 2006. Bodenbildung in entwässerten Mudden. Telma 36, 39-52.

Choiński, A., Ptak, M., Strzelczak, A., 2012. Examples of lake disappearance as an effect of reclamation works in Poland. Limnological Review 12(4), 161-167. https://doi.org/10.2478/v10194-012-0056-2

Ermert, O., 1963. Landeskultur in Ostpreussen. Schriftenreihe des Kuratoriums für Kulturbauwesen 10, 167-224.

Frank, S., Tiemeyer, B., Bechtold, M., Lucke, A., Bol, R., 2017. Effect of past peat cultivation practices on present dynamics of dissolved organic carbon. Science of the Total Environment 574, 1243-1253. https://doi. org/10.1016/j.scitotenv.2016.07.121.

Freytel, P., Verrecchia, P., 2002. Lacustrine and palustrine carbonate petrography: an overview. Journal of Paleolimnology 27, 221-237.

Gąsiorowski M., 2001. Lacustrine chalk deposition in Lake Kruklin (NE Poland) as a result of decalcification of the lake catchment. Studia Quaternaria 18, 17-24.

Ilnicki, P., 2002. Peatlands and peat (Torfowiska i torf). Wydawnictwo Akademii Rolniczej im. Augusta Cieszkowskiego w Poznaniu, Poznań, 606 pp. (in Polish).

IUSS Working Group WRB, 2015. World Reference Base for Soil Resources 2014, update 2015. International soil classification system for naming soils and creating legends for soil maps. World Soil Resources Report No. 106, FAO, Rome, Italy, 203 pp.

Jarnuszewski, G., Meller, E., 2018. Morphological and physical properties of dehydrated Holocene carbonate limnic deposits in post-bog areas of NW Poland. Journal of Ecological Engineering 19(1), 136-142. https://doi.org/10.12911/22998993/79412

Jarnuszewski, G., Meller, E., 2019. Total content of macroelements and trace elements in Holocene calcareous gyttja from the post-bog area of north-western Poland. Soil and Water Research 14(1), 40-46. https://doi.org/10.17221/146/2017-SWR

Kabała, C. et al., 2019. Polish Soil Classification, 6th edition - principles, classification scheme and correlations. Soil Science Annual 70(2), 71-97. https://doi.org/10.2478/ssa-2019-0009

Körnig, F., 1908. Bericht über die domänenfiskalischen Wiesenanlagen auf dem ehemaligen Dimmern-See im Kreise Ortelsburg. Mitteilungen des Vereins zur Förderung der Moorkultur im Deutschen Reiche (Berlin) 26(16), 260-268; 26(17), 278-282.

Kruczkowska, B., Jonczak, J., Słowińska, S., Bartczak, A., Kramkowski, M., Uzarowicz, Ł., Tyszkowski, S., Słowiński, M., 2021. Stages of soil development in the coastal zone of a disappearing lake-a case study from central Poland. Journal of Soils and Sediments 21, 1420-1436. https://doi.org/10.1007/s11368-021-02880-8

Lemkowska, B., Sowiński, P., 2018. Limnic Rendzinas in the Mazurian Lakeland (NE Poland). Soil Science Annual 69(2), 109-120. https://doi. org/10.2478/ssa-2018-0011

Lundquist, G., 1927. Bodenablagerungen und Entwicklungstypen der Seen. Die Binnengewässer, Vol. 2. E. Schweizerbartsche Verlagsbuchhandlung, Stuttgart, 1-124.

Łabaz, B., Kabała, C., 2016. Human-induced development of mollic and umbric horizons in drained and farmed swampy alluvial soils. Catena 139, 117-126. https://doi.org/10.1016/j.catena.2015.12.013

Łachacz, A., Nitkiewicz, M., Pisarek, W., 2009. Soil conditions and vegetation on gyttja lands in the Masurian Lakeland. [In:] Łachacz, A. 
(Ed.),Wetlands - their functions and protection. Department of Land Reclamation and Environmental Management, University of Warmia and Mazury in Olsztyn, 61-94.

Łachacz, A., Kalisz, B., 2016. Polish contribution to the study of moorshforming process. [In:] Łachacz, A., Kalisz, B. (Eds), Polish National Committee of International Peatland Society - history, activities, achievements. Wydawnictwo Uniwersytetu Warmińsko-Mazurskiego w Olsztynie, Olsztyn, 115-130.

Łachacz, A., Pisarek W., 2018. Selected plant species of wetlands in the first section of the Kanał Dymerski canal in the Mrągowo Lakeland (NE Poland). Fragmenta Floristica et Geobotanica Polonica 25(1), 65-78. (in Polish with English summary).

Markiewicz, M., Gonet, S. S., Marszelewski, W., Mendyk, Ł., Sykuła, M., 2017. Differentiation of soils and land use changes in the vicinity of the disappeared Gardeja lake (Northern Poland). Soil Science Annual 68(3), 115-124. https://doi.org/10.1515/ssa-2017-0014

Markowski, S., 1980. Structure and properties of peatlands' bottom lake sediments of frequent occurrence in West Pomerania region as a basis for their identification and classification. [In:] Post-conference materials "Lacustrine chalk and gyttjas" T. 2. PTPNoZ, Oddział Gorzów Wielkopolski-Zielona Góra, 45-55. (in Polish).

Meller, E., 2006. Shallow organogenic-calcareous soils on lacustrine chalk and their transformation resulted from cultivation. Akademia Rolnicza w Szczecine, Rozprawy 233, 1-115. (in Polish with English summary)

Mendyk, Ł., Markiewicz, M., Bednarek, R., Świtoniak, M., Gamrat, W. W., Krześlak, I., Sykuła M., Gersztyn, L., Kupniewska, A., 2016. Environmental changes of a shallow kettle lake catchment in a young glacial landscape (Sumowskie Lake catchment), North-Central Poland. Quaternary International 418, 116-131. http://dx.doi.org/10.1016/ j.quaint.2015.10.008

Mendyk, Ł., Świtoniak, M., Bednarek, R., Falkowski, A., 2015. Genesis and classification of the soils developed from the sediments of the former Oleszek mill pond basin (the Chełmińskie Lakeland, N Poland). Soil Science Annual 66 (1), 29-35. https://doi.org/10.1515/ssa-2015-0016

Okruszko, H., 1976. Rules of identification and repartition of hydrogenic soils in the view of their reclamation requirements. Biblioteczka Wiadomości IMUZ 52, 7-53. (in Polish).

Okruszko, H., Ilnicki, P., 2003. The moorsh horizons as quality indicators of reclaimed organic soils. [In:] Parent, L.-E., Ilnicki, P. (Eds), Organic soils and peat materials for sustainable agriculture. CRC Press, Boca Raton, 1-14.

Oyama, M., Takehara, H., 1992. Revised standard soil color charts. Fujihara Industry Co., Tokio, Japan.

Papierowska, E., Szatyłowicz, J., Kurzawski, G., Łachacz, A., 2009. Evaluation of the wetting angle of detritus gyttja. Roczniki Gleboznawcze - Soil Science Annual 60(4), 65-70. (in Polish with English summary)

Piaścik, H., Gotkiewicz, J., Łachacz, A., 2003. Bestandsaufnahme und Bewertung der Feuchtgebiete im nordöstlichen Polen für den Umweltschutz. Telma 33, 231-238.

Polish Soil Classification (Systematyka gleb Polski), 1989. Roczniki Gleboznawcze - Soil Science Annual 40(3/4), 1-150. (in Polish with English summary).

Polish Soil Classification (Systematyka gleb Polski), 2011. Roczniki Gleboznawcze - Soil Science Annual 62(3), 1-193. (in Polish with English summary).

Prusinkiewicz, Z., Noryśkiewicz, B., 1975. Geochemical and paleopedological aspects of the origin of lake chalk as the parent rock for northern Poland rendzinas. Acta Universitatis Nicolai Copernici, Nauki Matematyczno-Przyrodnicze 35, Geografia 11, 115-137. (in Polish with English summary).

PTG 2009. Particle size distribution and textural classes of soils and mineral materials - classification of Polish Society of Soil Science 2008.
Roczniki Gleboznawcze - Soil Science Annual 60(2), 5-16. (in Polish with English abstract)

Sauerbrey, R., Lehrkamp, H., Göbel, F., 2003. Rimpau'sche Moordammkulturen in Brandenburg. Telma 33, 121-132.

Säurich, A., Tiemeyer, B., Dettmann, U., Don, A., 2019. How do sand addition, soil moisture and nutrient status influence greenhouse gas fluxes from drained organic soils? Soil Biology and Biochemistry 135, 71-84. https://doi.org/10.1016/j.soilbio.2019.04.013

Solon, J., et al., 2018. Physico-geographical mesoregions of Poland: Verification and adjustment of boundaries on the basis of contemporary spatial data. Geographia Polonica 91(2), 143-170. https://doi. org/10.7163/GPol.0115

Systematyka gleb Polski, wyd. 6, 2019. Wydawnictwo Uniwersytetu Przyrodniczego we Wrocławiu, Instytut Nauk o Glebie i Ochrony Środowiska Uniwersytetu Przyrodniczego we Wrocławiu, Polskie Towarzystwo Gleboznawcze. Komisja Genezy, Klasyfikacji i Kartografii Gleb, Wrocław-Warszawa, ss. 292.

Stankevica, K., Vincevica-Gaile, Z., Klavins, M., 2016. Freshwater sapropel (gyttja): its description, properties and opportunities of use in contemporary agriculture. Agronomy Research 14(3), 929-947.

Świtoniak, M., Kabała, C., Charzyński, P., 2016. Proposal of English equivalents for the soil taxa names in the Polish Soil Classification. Soil Science Annual 67(3), 103-116. (in Polish with English summary) https://doi.org/10.1515/ssa-2016-0013

Tobolski, K., 2000. A guide for identifying peats and lacustrine deposits (Przewodnik do oznaczania torfów i osadów jeziornych). Wydawnictwo Naukowe PWN, Warszawa, 508 pp. (in Polish)

Uggla, H., 1962. Evolution and properties of several gyttja soils of the Masurian Lakeland. Zeszyty Naukowe WSR w Olsztynie 12(162), 265-274. (in Polish with German summary)

Uggla, H., 1964. The influence of the drainage area on the formation and some properties of the lake sediments. Zeszyty Naukowe WSR w Olsztynie 17(355), 645-654. (in Polish with English summary)

Uggla H., 1967. Über die Torf- und Gyttjamurschböden Polens. Wissenschaftliche Zeitschrift der Universität Rostock, 16, 105-115.

Uggla, H., 1968. Bog and mull soils of the gyttja moorland at Gązwa. Roczniki Gleboznawcze - Soil Science Annual 18(2), 369-414. (in Polish with English summary).

Uggla, H., 1969a. Gyttja soils of the Masurian Lakeland. I. General characteristic of gyttja-bog and gyttja-muck soils. Zeszyty Naukowe WSR w Olsztynie 25(3), 563-582. (in Polish with German summary)

Uggla, H., 1969b. Gyttja soils of the Masurian Lakeland. II. Physical, chemical and biological properties of gyttja-bog and gyttja-muck soils. Zeszyty Naukowe WSR w Olsztynie 25(3), 583-606. (in Polish with German summary)

Uggla, H., 1971. Characteristics of gyttja and gyttja soils of Mazurian Lakeland in light of hitherto investigations of the Chair of Pedology, College of Agriculture in Olsztyn. Zeszyty Problemowe Postępów Nauk Rolniczych 107, 14-25. (in Polish with English summary)

Uggla, H., 1976. Rendzinas in the Mazurian Lakeland. Roczniki Gleboznawcze - Soil Science Annual 27(2), 113-125. (in Polish with English summary)

Uggla, H., Mirowski, Z., 1968. Einfluss der Murschprozesse auf die Eigenschaften der Humusstoffe in Gyttjaböden. Roczniki Gleboznawcze - Soil Science Annual 19(dodatek), 149-161.

Uggla, H., Róg, Z., Wocławek, T., 1972. Micromorphology of gyttja muck soil of Jawty Małe. Zeszyty Problemowe Postępów Nauk Rolniczych 123, 481-489.

van Reeuwijk, L.P., (Ed.). 2002. Procedures for soil analysis. International Soil Reference and Information Centre, Technical Paper 9, Wageningen: 120 pp. 


\section{Klasyfikacja gleb wykształconych z osadów jeziornych w północno-wschodniej Polsce}

\section{Streszczenie}

Celem pracy było określenie przydatności najnowszej edycji Systematyki gleb Polski (2019) do odzwierciedlenia typowych cech gleb wykształconych z osadów jeziornych w porównaniu do wcześniejszych edycji tej systematyki (SGP, 1989, 2011) oraz do Word Reference Base for Soil Resources (WRB 2015). Na podstawie morfologii oraz właściwości przedstawiono pozycję taksonomiczną gleb w różnych klasyfikacjach. Z ogólnej liczby 25 profili glebowych, 18 wykształciło się przy różnym udziale osadów jeziornych, a 7 z różnorodnych materiałów zdeponowanych na brzegach byłych jezior. Na podstawie zawartości trzech głównych składników osadów dennych, a mianowicie materii organicznej określonej jako straty masy po prażeniu, węglanu wapnia oraz mineralnej masy bezwęglanowej, różne rodzaje materiałów limnicznych zostały zidentyfikowane w ramach SGP (2019). Gleby wykształcone z głębokich osadów jeziornych w ramach SGP (2019) zostały zaklasyfikowane jako gleby gytiowe, gdy nie podlegały procesowi murszenia, a do gleb gytiowych murszowych, gdy powierzchniowy poziom składał się z murszu gytiowego. Pewne problemy pojawiły się podczas klasyfikacji gleb wykształconych z płytkich pokładów gytii występujących na brzegach byłych jezior, których powierzchniowe poziomy wykształciły się przy udziale materiałów deluwialnych namytych z przyległych zboczy. W niektórych profilach powierzchniowy poziom wykształcił się z piasku celowo zdeponowanego przez człowieka na powierzchni gytiowiska w celu poprawy właściwości gleb. Na gytiowisku Łąki Dymerskie piaszczysty poziom powierzchniowy ma miąższość 31-39 cm, a dodatkowo są pisemne relacje na temat ulepszania tych gleb od 1885 roku za pomocą piaskowania (metoda Rimpau, Sanddeckkultur). Obecnie poziom ten zawiera 3-20\% SOM i spełnia kryteria sprecyzowane w SGP (2019) dla poziomu diagnostycznego arenimurszik. Najnowsza wersja Systematyki gleb Polski umożliwia precyzyjną klasyfikację takich gleb przez użycie odmiany - nasypowe. W ramach WRB (2015) takie gleby są zaliczane głównie do Hypereutric Rheic Drainic Histosols (Limnic, Nechic, Areninovic) lub do Eutric Gleysols (Endoarenic, Drainic, Humic, Limnic, Nechic). Ogólnie pozycja taksonomiczna badanych gleb jest lepiej oddana w SGP (2019) w stosunku do występowania różnorodnych warstw i różnych materiałów glebowych w porównaniu do wcześniejszych edycji Systematyki gleb Polski. Należy zauważyć, że precyzja SGP (2019) w stosunku do gleb gytiowych i gleb towarzyszących im w krajobrazie jest porównywalna do WRB (2015). 